First publ. in: Economics Letters 101 (2008), 2, pp. 134-136

\title{
Learning, bounded memory, and inertia ${ }^{\text {is }}$
}

\author{
Carlos Alós-Ferrer * \\ Department of Economics, University of Konstanz, Box 150, D-78457 Konstanz, Germany
}

\section{A B S T R A C T}

This paper considers bounded-memory players in a coordination game, who imitate the most successful remembered actions. With exogenous inertia, risk-dominant equilibria are selected independently of the length of memory. Without inertia, Pareto-dominant equilibria arise when memory is long enough.

\author{
Keywords: \\ Imitation \\ Inertia \\ Learning \\ Memory \\ Mutations \\ JEL classification: \\ C72 \\ D83
}

Consider a finite population of $N$ agents who recurrently play a symmetric, bilateral game sequentially against each other agent in the population (round-robin tournament). In a classical result, Kandori et al. (1993) (hereafter KMR) show that, if the bilateral game is a $2 \times 2$ coordination game, and the dynamics is such that strategies leading to the highest payoffs spread, then agents coordinate on risk-dominant equilibria in the long run, even in the presence of an alternative, Pareto-efficient equilibrium. This result gave rise to a large literature on learning in games which often builds on risk-dominance as the appropriate selection criterion. Our purpose is to perform a robustness test and show that, for a natural extension of the model, the selection result can be reversed.

The KMR model can be readily interpreted as a model of imitation (see KMR, p.31; Rhode and Stegeman, 1996; Sandholm, 1998) where agents mimic the actions which led to highest payoffs in the last period. In this note we consider exactly such a framework and endow agents with bounded memory, hence allowing them to make use of the information gained in the most recent periods of play. Agents remember all actions and payoffs observed in the last $K \geq 0$ periods of play in addition to the current one.

The imitation rule used in KMR can be described as "imitate the best", where simply the action leading to the highest payoff is

\footnotetext{
is Financial support from the Austrian Science Fund (FWF) under Project P18141-G09 is gratefully acknowledged.

* Tel.: +49 7531882340 . Fax: +49 7531884119.

E-mail address: Carlos.Alos-Ferrer@uni-konstanz.de.
}

mimicked. ${ }^{1}$ In a framework with memory, the rule will specify to imitate the action which has led to highest payoffs in remembered experience. $^{2}$

A standard element in learning models is the presence of exogenous inertia (see e.g. Samuelson, 1994 or Kandori and Rob, 1995), defined as an exogenously given probability $0 \leq \rho<1$ that each single agent is not able to adjust his strategy. We argue here that exogenous inertia counteracts the effects of memory, since there is positive probability that agents are denied revision opportunities and forced to stay with their current actions until relevant previous ones are forgotten.

Let the coordination game $G$ have payoff matrices given by

\begin{tabular}{ll|l|l|}
\hline \multicolumn{1}{c}{$P$} & \multicolumn{2}{c}{$P$} \\
\hline & $P$ & $a, a$ & $b, c$ \\
\hline & $R$ & $d, d$ \\
\cline { 2 - 3 } & &
\end{tabular}

such that $a>c, d>b, a>d$, and $a+b<c+d$. Hence, $(P, P)$ and $(R, R)$ are strict Nash equilibria, $(P, P)$ is Pareto efficient and $(R, R)$ is risk dominant. This is the most interesting case.

\footnotetext{
1 This rule is also used e.g. by Robson and Vega-Redondo (1996), Vega-Redondo (1997), Alós-Ferrer et al. (2000), and Alós-Ferrer and Weidenholzer (in press).

2 A natural criticism is that the action associated to the best payoff might have a worse average than other actions. Experiments in psychology, however, seem to indicate that human decision makers tend to select alternatives yielding high (salient) payoffs, even when those alternatives are associated with lower expected returns. This effect is called "underweighting of rare events" by Barron and Erev (2003) (see also Erev and Barron 2005). An analysis of more general imitation rules is beyond the scope of this note.
} 
Each period, agents play with each other in a round-robin tournament. Since there are only two strategies in the underlying bilateral game, the state space can be represented in a reduced form as $\Omega^{K+1}$ with $\Omega=\{0, \ldots, N\}$, where a typical coordinate $n$ is identified with the number of agents playing strategy $P$ in the corresponding period. We follow KMR and assume $N$ even for simplicity. ${ }^{3}$

Let $\Pi(P, n)$ and $\Pi(R, n)$ be the payoffs of an agent playing $P$ or $R$ when exactly $n$ agents are playing $P(\Pi(P, 0)$ and $\Pi(R, N)$ are not defined). That is,

$\Pi(P, n)=(n-1) a+(N-n) b \quad$ and $\quad \Pi(R, n)=n \cdot c+(N-n-1) d$.

After payoff realization, each agent $i=1, \ldots, N$ independently gets the opportunity to revise his strategy with probability $1-\rho>0$. Agents who can revise their strategies will do so according to the imitate-thebest rule with memory $K$. As in KMR, the model is completed with the possibility of rare mistakes or experiments. That is, each period each agent might tremble with probability $\varepsilon>0$, independent across periods and agents, thereafter choosing a strategy at random. We refer the reader to Ellison (2000) for an exposition on this class of "vanishing mutation" models. The focus is on stochastically stable states, which are those in the support of the limit invariant distribution of the process as $\varepsilon \rightarrow 0$. The process where $\varepsilon=0$ is called the unperturbed dynamics.

If agents use the imitate-the-best rule with bounded memory of length $K$, the KMR model can be taken as the particular case with $K=0$. Further, the general case allows for easy comparison of the results with and without exogenous inertia and memory.

Note that the dynamics and its unperturbed version are Markov chains. First we claim that, for all $0 \leq \rho<1$ and all $K \geq 0$, the only absorbing sets of the unperturbed dynamics are the singletons $\{(0, \ldots+1,0)\}$ and $\{(N, .+\ldots+1, N)\}$. Hence, the two Nash equilibria are the only candidates for stochastic stability.

To see this, first note that these two states are absorbing, since there is probability one of remaining in them once they are reached (only one strategy is remembered, thus it is the only one which can be imitated). Second, any other state $\omega$ must be transient. It is enough to show that there exists a positive-probability path starting at $\omega$ and finishing in one of the two states above.

Since $\rho<1$, with positive probability (w.p.p.) all agents will receive revision opportunities during $K+1$ periods. W.p.p. each period $t$, all of them imitate the same strategy as other agents, thus reaching a state of the form $\left(n_{0}, \ldots, n_{K}\right)$ where $n_{t} \in\{0, N\}$ for all $t=0, \ldots, K$. If all $n_{t}=0$, the argument is complete. If not, the maximum payoff remembered is that of $P$ in a profile where all players simultaneously play $P$. The following period, w.p.p. all agents choose $P$, receiving the maximum payoff again. Since this profile and the associated payoff will be remembered for the next $K+1$ periods, agents will again choose $P$ w.p.p. until the state $(N, \ldots, N)$ is reached.

KMR show that their dynamics (without memory) selects the riskdominant equilibrium. Their result translates to our framework as follows. For $K=0$ and any $\rho>0$, the only stochastically stable state of the dynamics is the singleton $\{0\}$, i.e. the risk-dominant equilibrium. This result arises from the fact that, without memory, the Paretoefficient outcome is easy to destabilize, in spite of having strictly larger payoffs. Once a mutation occurs, the payoffs of the Pareto-efficient equilibrium are no longer observed. Neither are they remembered. It seems plausible, though, that agents would not forget strictly superior payoffs immediately. This intuition is captured by setting $K>0$.

We now present the result. We say that $a \in\{R, P\}$ is a stochastically stable strategy if the corresponding state where all players adopt $a$ for $K+1$ periods is stochastically stable.

\footnotetext{
${ }^{3}$ Rhode and Stegeman (1996) drop this requirement, showing that in some extreme cases, with $N$ odd, the predictions of KMR may change.
}

\section{Theorem 1. Let $G$ be as above and $N$ even.}

(a) Let $\rho=0$. For $K$ large enough, ${ }^{4}$ the only stochastically stable strategy is $P$, i.e. the Pareto-efficient equilibrium is selected.

(b) For any $\rho>0$ and $K \geq 0$, the only stochastically stable strategy is $R$, i.e. the risk-dominant equilibrium is selected.

Proof. Let $c(R, P)$ and $c(P, R)$ be the minimum number of mutations needed to reach the state $(N, \ldots, N)$ from $(0, \ldots, 0)$ and vice versa. Since the corresponding singletons are the only absorbing sets of the unperturbed dynamics, by a standard argument $R$ is stochastically stable if and only if $c(R, P) \geq c(P, R)$, and vice versa.

Consider a profile where exactly $n$ agents play $P$. The difference in payoffs between $P$ - and $R$-players is $\Pi(P, n)-\Pi(R, n)$. A straightforward computation shows that, for $1 \leq n \leq N-1, \Pi(P, n)-\Pi(R, n)$ is increasing in $n$. Moreover, $\Pi(P, n)=\Pi(R, n)$ if and only if $n=n^{*}$, where

$n^{*}=\frac{N(d-b)+(a-d)}{(a-c)+(d-b)}>\frac{N}{2}$

Hence, $P$-players obtain lower payoffs than $R$-players if and only if $n \leq n^{*}$. In particular, since $n^{*}>\frac{N}{2}, \Pi(P, n)-\Pi(R, n)<0 \forall n \leq \frac{N}{2}$.

To prove (a), let $\rho=0$ and $K>0$. We want to show that $c(R, P)<c(P, R)$. Consider first the state $(0, \ldots, 0)$. If $n$ simultaneous experiments (with $P$ ) occur, three payoffs will be observed and remembered: $\Pi(R, n)$, $\Pi(P, n)$, and $\Pi(R, 0)$. By Eq. (1), $\Pi(P, n)-\Pi(R, n) \geq 0 \Leftrightarrow n \geq n^{*}$. Analogously,

$\Pi(P, n)-\Pi(R, 0) \geq 0 \Leftrightarrow n \geq \frac{N \cdot(d-b)+(a-d)}{(a-b)}=: \hat{\mathrm{n}}$

Thus, $\max \left(n^{*}, \hat{\mathrm{n}}\right)$ simultaneous experiments suffice for the transition from $(0, \ldots, 0)$ to $(N, \ldots, N)$ (if this is larger than $N, N$ experiments suffice). This yields the bound $c(R, P) \leq\left[\max \left(n^{*}, \hat{\mathrm{n}}\right)\right]^{5}$ which is independent of K.

Consider now the state $(N, \ldots, N)$. If $N-n$ experiments (with $R$ ) occur, three payoffs will be observed and remembered: $\Pi(R, n), \Pi(P, n)$, and $\Pi(P, N)$. However, it is immediate to see that $\Pi(P, N)>\Pi(R, n)$ for all $n$. Hence, after any number of experiments, all agents will return to strategy $P$. The transition from $(N, \ldots, N)$ to $(0, \ldots, 0)$ cannot occur as long as the profile where all agents were playing $P$ is remembered. Moreover, agents will immediately revert to this profile in the absence of further experiments.

It is thus clear that any successful transition from $(N, \ldots, N)$ to $(0, \ldots, 0)$ must involve experiments during $K+1$ consecutive periods, i.e. until the payoff $\Pi(P, N)$ is forgotten. This means that $c(P, R) \geq K+1$.

In summary, $c(P, R) \geq K+1$ and $c(R, P) \leq\left[\max \left(n^{*}, \hat{\mathrm{n}}\right)\right]$. For $K$ long enough, the first is larger, hence $(N, \ldots, N)$ becomes the only stochastically stable state, i.e. the Pareto-efficient equilibrium is selected.

We now turn to (b). Let $\rho>0$ and $K \geq 0$. Consider any state $\omega=\left(n_{-K}, \ldots\right.$, $\left.n_{-1}, n_{0}\right)$ with $n_{t} \leq \frac{N}{2} \forall t=-K, \ldots, 0$. In such a state agents will never imitate $P$. Further, when given the opportunity, the $P$-players will switch to $R$. An immediate implication is that $c(R, P)>\frac{N}{2}$.

It suffices now to show that $c(P, R) \leq \frac{N}{2}$. This will be proved if we exhibit an appropriate positive-probability transition involving only $\frac{N}{2}$ experiments.

Consider the state $(N, \ldots, N)$. Suppose exactly $\frac{N}{2}$ experiments with $R$ occur. W.p.p., no agent receives revision opportunity for $K$ consecutive periods. After those $K$ periods, the process is in the state $\left(\frac{N}{2}, \ldots, \frac{N}{2}\right)$, and there are only two payoffs in the agents' memories: $\Pi\left(P, \frac{N}{2}\right)$ and $\Pi\left(R, \frac{N}{2}\right)$. Since, by Eq. (1), $\Pi\left(P, \frac{N}{2}\right)<\Pi\left(R, \frac{N}{2}\right)$, if now all the agents receive revision opportunities, they will imitate $R$. If $K=0$, the transition is complete. If $K>0$, there are three payoffs in the agents' memories, one for $P$ (namely

\footnotetext{
${ }^{4}$ It is possible to show that the required length of memory is decreasing in population size.

${ }^{5}$ This maximum is equal to $n^{*}$ if $c>d$ and to $n$ if $c<d$.
} 
$\left.\Pi\left(P, \frac{N}{2}\right)\right)$ and two for $R\left(\Pi\left(R, \frac{N}{2}\right)\right.$ and $\left.\Pi(R, 0)\right)$, with at least one of the payoffs for $R$ larger than the payoff for $P$. Irrespective of revision opportunities, agents will keep choosing $R$ for $K$ more periods, completing the transition to $(0, \ldots, 0)$. In summary, $c(P, R) \leq \frac{N}{2}<c(R, P)$ and the result follows.

This result can be given two different interpretations. Focusing on part (a), one could argue that the introduction of memory changes the long-run predictions in learning models for games, as long as there is no exogenous inertia. Indeed, part (a) shows that bounded memory reverses the standard equilibrium selection result in coordination games, and thus that result is not robust. The reason is that memory allows agents using the imitate-the-best rule to behave as if they were able to experiment conditionally. When an experimenter tries out a new strategy, he will observe its success relative to the payoffs obtained the previous period. If the experiment brings payoffs down, the experimenter will be able to correct his mistake and go back to the previous action. This observation, which can be interpreted as trial-and-error learning, is of intertemporal nature in an explicitly dynamic framework, and naturally introduces better-response considerations into models of bounded rationality without explicitly assuming that the agents are able to compute best replies. In the case of coordination games, Theorem 1 shows that the equilibrium selection result of KMR changes as the memory length increases. This is in line with the intuition that imitation with bounded memory favors high-payoff outcomes, even in models where agents are extremely naive. ${ }^{6}$

If we focus on part (b) instead, we can defend a different interpretation of Theorem 1. Part (b) illustrates that, in the presence of any fixed, positive level of exogenous inertia (no matter how small), the selection of riskdominant equilibria is robust to the introduction of bounded memory. Memory can have an effect only if inertia is totally suppressed. The reason is that exogenous inertia, no matter how small, counteracts the effects of memory. The transitions in the proof are constructed bypassing memory. Agents' desired adjustments are just blocked through inertia until previous knowledge disappears. Perhaps unreasonably, agents who have just revised their strategies are repeatedly denied the chance to review the consequences of the revision. This makes the interpretation of the experimentation process as trial and error problematic.

The assumption of exogenous inertia builds into the agents' behavior a hardwired preference to keep on doing what they did in the past, irrespective of payoffs. In the present paper, though, agents are assumed to imitate those actions which yield larger payoffs, among those that they observe and remember. Once the available set of actions is enlarged to include past ones, the assumption of memory simply introduces in the agents' behavior a preference to keep on doing what worked in the past. Exogenous inertia, modeled by an exogenous parameter $\rho>0$, simply eliminates this preference by undiscriminatingly favoring all past actions, irrespectively of whether they worked or not.

This observation ("to keep on doing what worked in the past") shows that, even with $\rho=0$, the current model incorporates a certain idea of inertia. The difference is that this kind of inertia is then an endogenous result, rather than an exogenous behavioral assumption.

It is worth examining the motivation for the introduction of inertia in the literature. Samuelson (1994, p.39) mentions that, in a best-reply framework, (exogenous) inertia "may be applicable in situations where calculating optimal actions is costly, difficult, or time-consuming, so that agents will usually simply repeat the actions they have taken in the past." It might be argued that reverting to a previously used action is neither costly nor difficult, and certainly not timeconsuming. The argument, however might be useful to interpret imitation-linked, endogenous inertia in favor of actions that have performed well in the past.

The complete removal of exogenous inertia is, however, not strictly necessary. The technical problem arising between inertia and memory

\footnotetext{
${ }^{6}$ See Alós-Ferrer and Weidenholzer (in press) for a different take on this intuition in
} a local interactions model. reduces to the following simple observation. When studying limit behavior as the experimentation probability $\varepsilon$ goes to zero, any behavior postulated with a fixed probability, such as exogenous inertia with probability $\rho$, becomes arbitrarily more probable than experimentation. In a sense, modeling inertia through an exogenous, constant parameter $\rho>0$ exaggerates its role. An alternative would be to study also the limit behavior as inertia becomes small, for example by linking its probability to the probability of experimentation.

Consider the following simple variant of the model. Each period, each agent has a probability of experimentation $\varepsilon$. For the sake of concreteness, assume that, after a tremble, an agent chooses the strategy opposite to the one he was previously using with probability $0<\theta<1$, and the same one with probability $1-\theta$. Further, each agent has a probability $\nu \varepsilon$ of upholding his strategy, where $\nu>1$. Let $\varepsilon$ be small enough so that $\varepsilon<\frac{1}{1+v}$. With the remaining probability, the agent follows the imitate-the-best rule with memory $K$. We refer to this variant as the model with vanishing inertia.

Proposition 1. The stochastically stable states in the model with vanishing inertia are identical to the ones of the basic model with $\rho=0$, i.e. the Pareto-efficient equilibrium is selected for K large enough.

Proof. The stochastically stable states in any vanishing mutations model are independent of the distribution with which strategies are selected after a mistake, conditional on a mistake happening, as long as that distribution has full support. In particular, the distribution can be agent- and state-dependent. ${ }^{7}$

The model with vanishing inertia can be rewritten as follows. There is no exogenous inertia, i.e. $\rho=0$. Each period, each agent has a probability $\varepsilon^{\prime}=(1+\nu) \varepsilon$ of experimentation. If experimenting, the agent will sample the strategy opposite to the one he chose previously with probability $\frac{\theta}{1+v}$ and the same one with the complementary probability. This model falls under case (a) of Theorem 1 and its transition probabilities are identical to those in the vanishing inertia case, hence the result follows.

In a sense, in the model with vanishing inertia, we simply reinterpret a mutation to the previously used strategy as inertia. Making the constant $\nu$ arbitrarily high (but fixed) allows to emphasize this behavior, without affecting the limiting results.

\section{References}

Alós-Ferrer, C. and Weidenholzer, S. (in press): "Contagion and Efficiency," Journal of Economic Theory.

Alós-Ferrer, C., Ania, A.B., Schenk-Hoppé, K.R., 2000. An evolutionary model of Bertrand oligopoly. Games and Economic Behavior 33,1-19.

Barron, G., Erev, I., 2003. Small feedback-based decisions and their limited correspondence to description-based decisions. Journal of Behavioral Decision Making 16 215-233.

Ellison, G., 2000. Basins of attraction, long-run stochastic stability, and the speed of step-by-step evolution. Review of Economic Studies 67, 17-45.

Erev, I., Barron, G., 2005. On adaptation, maximization, and reinforcement learning among cognitive strategies. Psychological Review 112, 912-931.

Kandori, M., Rob, R., 1995. Evolution of equilibria in the long run: a general theory and applications. Journal of Economic Theory 65, 383-414.

Kandori, M., Mailath, G.J., Rob, R., 1993. Learning, mutation, and long run equilibria in games. Econometrica 61, 29-56.

Rhode, P., Stegeman, M., 1996. A comment on "Learning, mutation, and long-run equilibria in games". Econometrica 64, 443-449.

Robson, A.J., Vega-Redondo, F., 1996. Efficient equilibrium selection in evolutionary games with random matching. Journal of Economic Theory 70, 65-92.

Samuelson, L., 1994. Stochastic stability in games with alternative best replies. Journal of Economic Theory 64, 35-65.

Sandholm, W.H., 1998. Simple and clever decision rules for a model of evolution. Economics Letters 61, 165-170.

Vega-Redondo, F., 1997. The evolution of Walrasian behavior. Econometrica 65, 375-384.

\footnotetext{
7 This is a well-known fact, which follows simply from the observation that limit rates are unaffected by the mentioned distribution. See e.g. the derivations of stochastic stability in either Kandori et al. (1993) or Ellison (2000).
} 